\title{
Safety of the Influenza A (H1N1)2009 Vaccine in Chronic Obstructive Pulmonary Disease: A Matched Case-Control Study
}

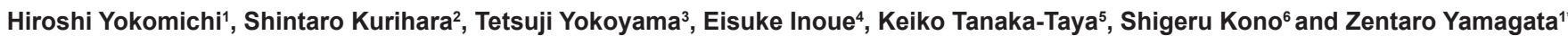

${ }^{1}$ Department of Health Sciences, Interdisciplinary Graduate School of Medicine and Engineering, University of Yamanashi, 1110 Shimokato, Chuo City, Yamanashi, Japan ${ }^{2}$ Infection Control and Education Center, Nagasaki University, 7-1 Sakamoto-1, Nagasakishi, Japan

${ }^{3}$ Department of Health Promotion National Institute of Public Health, 2-3-6 Minami, Wako, Saitama, Japan

${ }^{4}$ Department of Clinical Medicine (Biostatistics), School of Pharmacy, Kitasato University, 5-9-1 Shirokane, Minato-ku, Tokyo, Japan

5 Infectious Disease Surveillance Center, National Institute of Infectious Diseases, 1-23-1 Toyama Shinjuku-ku, Tokyo, Japan

${ }^{6}$ Second Department of Internal Medicine, Nagasaki University School of Medicine, 7-1 Sakamoto-1, Nagasakishi, Japan

\begin{abstract}
Background: The safety of influenza A (H1N1) 2009 among chronic obstructive pulmonary disease (COPD) patients has not been investigated yet. Our objective was to investigate the safety of the A (H1N1) 2009 vaccine, especially for mortality after vaccination.

Methods: From October 2009 to March 2010, we collected records from all Japanese hospitals registered in the Japanese Respiratory Society. We conducted a 1:1 matched case-control study. Patients with COPD who died in the study period were determined as case group patients $(n=36)$. Patients with COPD who survived the study period were determined as control group patients $(n=36)$. In the control group selection process, we selected age, sex, period of home oxygen therapy (measure for COPD severity), hospital, and calendar time as matching factors. We then compared the proportion of patients who received the influenza A (H1N1) 2009 vaccine in each group and evaluated the conditional odds ratio.
\end{abstract}

Results: Both the case and control groups had 36 COPD patients, 32 of whom were men. The mean age was 76.6 years $(S D=8.6)$ in the case group and 76.9 years $(S D=8.3)$ in the control group. The mean period of home oxygen therapy in both groups was 1.8. The proportion of patients receiving A (H1N1) 2009 vaccinations was $47.2 \%$ in the case group and $63.9 \%$ in the control group. The crude conditional odds ratio of mortality in the winter was 0.33 (95\% confidence interval: $0.06-1.34)$ and adjusted conditional odds ratio was 0.37 (95\% confidence interval: 0.09-1.52) with no significance.

Conclusions: Our study detected no statistically increased risk of mortality after influenza A (H1N1) 2009 vaccination among COPD patients. The results are, however, limited by the small sample size and low statistical power. A similar larger-scale study is needed in the future to confirm our findings.

Keywords: Influenza vaccines; Vaccine safety; Influenza A Virus, H1N1subtype; Chronic obstructive pulmonary disease; Mortality; Case-control studies; Vaccines; Influenza, Human

Abbreviations: COPD - Chronic Obstructive Pulmonary Disease; SD - Standard Deviation

\section{Introduction}

Since the novel influenza A (H1N1) 2009 virus was identified in Mexico and the United States in April 2009, it has spread worldwide. In response to this situation, the World Health Organization raised the pandemic alert level to phase 6 [1]. Since seasonal influenza vaccines were not expected to prevent influenza A (H1N1)2009 virus infection [2], the development of efficient and safe new influenza vaccines was an urgent need.

The suspected side effects of the seasonal influenza vaccine include acute disseminated encephalomyelitis and Guillain-Barre syndrome [3-8]. After the dissemination of an estimated 82.4 million doses of A(H1N1)2009 vaccines, 48 fatal cases were reported to the Vaccine Adverse Event System in United States [6]. When the new influenza A (H1N1) 2009 spread in Japan, beginning in September 2009, the new vaccines were administered to the Japanese population. One hundred thirty-one patients with underlying diseases, including 22 with chronic kidney diseases, 12 with chronic obstructive pulmonary diseases (COPD), and 11 interstitial pneumonias, were reported to have died after the vaccinations $[9,10]$. The cause of death was undetermined between adverse events resulting from the vaccinations, the effects of the underlying diseases, or exacerbation of the underlying diseases by the vaccination. To our knowledge, thus far, no studies have tested the safety of the influenza vaccine among COPD patients.

To clarify the mortality risk associated with the vaccinations, we undertook a case-control study in which the patients who died with COPD (case) were matched with those who survived COPD (control). Patients with incurable COPD usually consult a respiratory physician throughout their lives in hospitals registered with the Japanese Respiratory Society in Japan. Our study was designed to determine the safety of the influenza A (H1N1) 2009 vaccine among COPD patients [10]. We aimed at exploring whether the A (H1N1) 2009 vaccine increased the mortality in patients with COPD.

*Corresponding author: Zentaro Yamagata, Department of Health Sciences, Interdisciplinary Graduate School of Medicine and Engineering, University of Yamanashi, 1110 Shimokato, Chuo City, Yamanashi, Japan, Tel: +81 55273 9566; Fax: +81552737882; E-mail: zenymgt@yamanashi.ac.jp

Received August 03, 2012; Accepted September 13, 2012; Published Septembe 15, 2012

Citation: Yokomichi H, Kurihara S, Yokoyama T, Inoue E, Tanaka-Taya K, et al. (2012) Safety of the Influenza A (H1N1)2009 Vaccine in Chronic Pulmonary Obstructive Disease: A Matched Case-Control Study. J Vaccines Vaccin 3:148. doi:10.4172/2157-7560.1000148

Copyright: (c) 2012 Yokomichi H, et al. This is an open-access article distributed under the terms of the Creative Commons Attribution License, which permits unrestricted use, distribution, and reproduction in any medium, provided the original author and source are credited. 
Citation: Yokomichi H, Kurihara S, Yokoyama T, Inoue E, Tanaka-Taya K, et al. (2012) Safety of the Influenza A (H1N1)2009 Vaccine in Chronic Pulmonry Obstructive Disease: A Matched Case-Control Study. J Vaccines Vaccin 3:148. doi:10.4172/2157-7560.1000148

Page 2 of 4

\section{Methods}

\section{Case definition and control selection}

COPD is generally diagnosed by a respiratory physician according to the guidelines published by The Japanese Respiratory Society. The case group included patients aged 18 or above who had been diagnosed with COPD, received home oxygen therapy (HOT), and died during the study period (October 1, 2009 to March 31, 2010), regardless of the cause of death. In this matched case-control study, for each case (dead) COPD patient, we selected 1 control (surviving) COPD patient in the same hospital on the same day when the selected case control group patient died ("index date") [11]; the pairs of case group and control group (i.e. dead and surviving) patients were matched according to sex, age ( \pm 3 years), and the HOT period ( \pm 2 years). COPD gradually increases in severity along with the HOT period [12], and the control patient had to be at the same risk of death as the case patient. We, therefore, used the HOT period as an available standard to determine the COPD severity, and we matched the case and control pair according to the HOT period on the index date. If more than 1 control patient matched a case patient in the same hospital on the index date, the control patient who was first in terms of alphabetical order of their last names was chosen. Thus, all necessary information about a case-control pair was collected from clinical records on the index date. Moreover, information on the control after the index date was not used in this study [11].

\section{Exposure to vaccine}

Information on whether a patient was administered to $0.5 \mathrm{~mL}$ of influenza A (H1N1) 2009 vaccine (a monovalent, inactivated vaccine with $15 \mu \mathrm{g}$ hemagglutinin per $0.5 \mathrm{~mL}$ content without adjuvant) prior to the index date was reported by the physician and was, thus, available in the clinical record.

\section{Covariates}

Information on 2 covariates considered to be confounders was gathered simultaneously from clinical records. The covariates identified were seasonal influenza vaccination records in that winter and the presence of diabetes mellitus. The history of seasonal influenza vaccination might indicate that the patient tended to be administered the vaccination by the respiratory physician. The history of diabetes mellitus might also have been a confounder that affected the COPD severity, as steroids are usually administered to COPD patients and can trigger drug-induced diabetes. Thus the results were adjusted for these 2 covariates.

\section{Statistical analysis}

We estimated the crude conditional odds ratio (OR) and its 95\% confidence interval (CI) to determine the association between A (H1N1) 2009 vaccination and mortality. Subsequently, using conditional logistic regression [13], we estimated the conditional ORs and their CIs, conditioned by age, sex, and HOT period, after adjusting for diabetes mellitus as a complication and history of seasonal influenza vaccination. For small-sample studies, the exact permutation test provides more accurate values of $\mathrm{OR}$ and $\mathrm{CI}$ than the conventional conditional logistic regression method; therefore, we used the exact permutation test. We used 95\% CIs instead of two-sided $\mathrm{p}$ values because they are more informative than the latter. In addition, we conducted 2 sensitivity analyses as follows: (1) in one, the primary outcome was not death from all causes but was restricted to death due to pneumonia, COPD exacerbation, or cardiac failure (26 pairs); (2) in

\begin{tabular}{|c|c|c|}
\hline & Case group & Control group \\
\hline Male/total & \multicolumn{2}{|c|}{$32 / 36(88.9 \%)$} \\
\hline Age & $76.6\left(\mathrm{SD}^{\mathrm{a}} 8.6\right)$ & $76.9\left(\mathrm{SD}^{\mathrm{a}} 8.3\right)$ \\
\hline HOT period (year) & $1.8\left(\mathrm{SD}^{\mathrm{a}} 2.0\right)$ & $1.8\left(\mathrm{SD}^{\mathrm{a}} 2.1\right)$ \\
\hline $\mathrm{A}(\mathrm{H} 1 \mathrm{~N} 1) 2009$ vaccination & $17 / 36(47.2 \%)$ & $23 / 36(63.9 \%)$ \\
\hline Seasonal influenza vaccination & $5 / 32(15.6 \%)$ & $9 / 33(27.3 \%)$ \\
\hline Incidence of influenza & $0 / 32(0.0 \%)$ & $0 / 33(0.0 \%)$ \\
\hline Diabetes mellitus & $2 / 34(5.9 \%)$ & $4 / 35(11.4 \%)$ \\
\hline Current smoking & $1 / 34(2.9 \%)$ & $1 / 35(2.9 \%)$ \\
\hline
\end{tabular}

aStandard deviation

Table 1: Characteristics of the patients

\begin{tabular}{|l|c|}
\hline \multicolumn{1}{|c|}{ Cause of mortality } & Cases \\
\hline Pneumonia & $14(38.9 \%)$ \\
\hline Exacerbation of COPD & $9(25.0 \%)$ \\
\hline Cardiac failure & $4(11.1 \%)$ \\
\hline Lung cancer & $2(5.6 \%)$ \\
\hline Acute myocardial infarction & $1(2.8 \%)$ \\
\hline Rupture of aortic aneurysm & $1(2.8 \%)$ \\
\hline Sepsis & $1(2.8 \%)$ \\
\hline Renal failure & $1(2.8 \%)$ \\
\hline Unknown & $3(8.3 \%)$ \\
\hline Total & $36(100 \%)$ \\
\hline
\end{tabular}

a Chronic obstructive pulmonary disease

Table 2: Causes of mortality in the case group.

\begin{tabular}{|l|c|c|}
\hline \multicolumn{1}{|c|}{ Reason for non-vaccination } & Case group & Control group \\
\hline Shortage of A(H1N1)2009 vaccine & $5(33.3 \%)$ & $1(11.1 \%)$ \\
\hline Poor physical condition & $4(26.7 \%)$ & $0(0.0 \%)$ \\
\hline Use of steroids & $0(0.0 \%)$ & $1(11.1 \%)$ \\
\hline Patient's refusal & $5(33.3 \%)$ & $6(66.7 \%)$ \\
\hline Unknown & $1(6.7 \%)$ & $1(11.1 \%)$ \\
\hline Total & $15(100 \%)$ & $9(100 \%)$ \\
\hline
\end{tabular}

Table 3: Reasons why patients did not receive $A(H 1 N 1) 2009$ vaccination.

the second, the case group patients were restricted to those who were not in poor physical condition nor in shortage of vaccines (27 pairs).

All statistical analyses were performed using SAS version 9.3 (Cary, NC, USA).

This study protocol was approved by the Ethics Review Committee of the Faculty of Medicine, University of Yamanashi, according to the regulations of the Declaration of Helsinki. Informed consent was not required for the study.

\section{Results}

\section{Participant characteristics}

We received patient information from 110 hospitals and enrolled 36 case-control pairs in the study. Of the 36 pairs of enrolled case-control patients, 32 pairs were of men. The mean age was $76.6(\mathrm{SD}=8.6)$ in the case group and $76.9(\mathrm{SD}=8.3)$ in the control group. The mean HOT period was $1.8(\mathrm{SD}=2.0)$ years in the case group and $1.8(\mathrm{SD}=2.1)$ years in the control group. Of the 36 patients in each group, 17 (47.2\%) in the case group and 23 (63.9\%) in the control group were vaccinated. Data on the history of seasonal influenza vaccination, infection of influenza, history of diabetes mellitus, and smoking statuses are shown in table 1. Among the causes of mortality in the case group, pneumonia was the most frequent, followed by exacerbation of COPD (Table 2). Table 3 lists the reasons why physicians did not vaccinate patients in either group. For the case group, the most common reason was shortage of 


\begin{tabular}{|l|c|c|c|c|}
\hline Conditional OR (pairs) & $\begin{array}{c}\text { Crude OR } \\
(\mathrm{Cl})\end{array}$ & Model 1 & Model 2 & Model 3 \\
\hline $\begin{array}{l}\mathrm{A}(\mathrm{H} 1 \mathrm{~N} 1) 2009 \\
\text { vaccination (36) }\end{array}$ & $\begin{array}{c}0.33 \\
(0.06-1.34)\end{array}$ & $\begin{array}{c}0.43 \\
(0.07-1.81)\end{array}$ & $\begin{array}{c}0.31 \\
(0.05-1.26)\end{array}$ & $\begin{array}{c}0.37 \\
(0.09-1.52)\end{array}$ \\
\hline $\begin{array}{l}\text { Seasonal influenza } \\
\text { vaccination (32) }\end{array}$ & $\begin{array}{c}0.43 \\
(0.07-1.88)\end{array}$ & $\begin{array}{c}0.47 \\
(0.11-1.95)\end{array}$ & - & $\begin{array}{c}0.44 \\
(0.10-1.85)\end{array}$ \\
\hline Diabetes mellitus (33) & $\begin{array}{c}0.50 \\
(0.05-3.49)\end{array}$ & - & 0.36 & 0.43 \\
$(0.06-2.37)$ & $(0.06-3.34)$ \\
\hline
\end{tabular}

Table 4: Crude conditional mortality odds ratio, adjusted conditional mortality odds ratio, and their confidence intervals (Cls). Odds ratios (ORs) were calculated for the case-control pairs.

vaccines and refusal by the patient. Refusal by the patient was a more common reason in the control group than it was in the case group.

\section{Odds ratios}

The proportion of vaccinations was lower in the case group than in the control group, indicating that the mortality was lower in the vaccinated patients than in the non-vaccinated patients (crude conditional $\mathrm{OR}=0.33,95 \% \mathrm{CI}=0.06-1.34)$. The ORs were adjusted for the history of seasonal influenza vaccination and diabetes mellitus (Table 4). The sample size was not large enough to determine the relation between A (H1N1) 2009 vaccine and mortality in COPD patients, if it existed. The sensitivity analyses showed almost the same results: (1) when the outcome was restricted to deaths due pneumonia, exacerbation, or cardiac failure, the crude conditional OR was 0.38 (95\% CI $=0.06-1.56)$ and adjusted conditional OR in Model 3 (Table 4) was 0.35 (95\% CI $=0.04-1.81)$; (2) when the case group was restricted to those who were not in poor physical condition nor experienced a shortage of vaccines, crude conditional OR was 0.60 (95\% CI $=0.09$ 3.08 ) and the adjusted conditional OR in Model 3 (Table 4) was 0.89 $(95 \% \mathrm{CI}=0.13-6.25)$.

\section{Discussion}

To our knowledge, this is the first study to investigate the safety of influenza A (H1N1) 2009 vaccines among patients with COPD, but not the first to examine the safety of the vaccine in the general population [14-20]. All the patients were of Japanese and mongoloid origin. The strengths of the study were that the case group patients and control group patients were matched for age, sex, HOT period, hospital, and calendar time as these factors were thought to be highly influential confounders. Therefore, both groups had similar features and were at an almost similar risk for death. Our results indicate that the influenza A (H1N1) 2009 vaccination does not increase the mortality risk of COPD patients. Such previous studies were not designed to examine the mortality [14-17]. We adjusted for history of seasonal influenza vaccination and diabetes mellitus, as diabetes mellitus is sometimes acquired if COPD has progressed. The conditional OR was 0.37 (95\% $\mathrm{CI}=0.09-1.52$ ).

Nakada et al. reported the possibility of fatal adverse events with underlying diseases after A (H1N1) 2009 vaccination [18]. However, this result was based on passive surveillance data. The safety of the A (H1N1) 2009 vaccine was not determined [19-21]. The Vaccine Adverse Event Reporting System in the United States reported 13 deaths after 46.2 million doses of A (H1N1)2009 vaccine were administered in the United States from October 5 to November 20, 2009. Of the 13 patients that died, 9 had a severe systemic disease and 1 died from a traffic accident [14]. After the post-marketing surveillance for adverse events resulting from 89.6 million doses of influenza A (H1N1)2009 vaccine administered in China, the Center for Disease Control reported 10 sudden deaths after administration. Nine of these patients had cardiovascular disease, liver failure, or stroke followed by cerebral herniation, and 1 patient, died 43 hours after vaccination and had no history of any medical condition [15]. A randomized clinical trial (RCT) in China involving 12,691 participants investigated the safety and immunogenicity of A (H1N1)2009 vaccines and found local reactions (pain, swelling, redness, etc.) and systemic reactions (fever, headache, myalgia, etc.) [16], but no instances of death occurring. Another RCT of 355 participants in Hungary also investigated the safety and immunogenicity of the vaccines and likewise, did not report any cases of death [17]. These 4 recent studies indicate that the A (H1N1)2009 influenza vaccination increases the mortality risk to only a slight extent, but the causes of mortality have not been determined. Moreover, the vaccine ought to have been effective and protected patients with underlying diseases from an influenza A (H1N1)2009 infection.

Our study has a few limitations. First, the sample size in our study was small. The COPD patients enrolled included both inpatients and outpatients, and we could not enroll sufficient numbers of casecontrol pairs belonging to the same hospital. Second, the control group population, on an average, might not have been in the same poor physical condition as the case group, nor have the same chance of access to vaccines as the case group population. We consider that the analysis adjusted for 2 covariates (Model 3 in Table 4) or the second sensitivity analysis compensated this limitation. Third, the study data depended on physician's reports, which were based on clinical records. However, since patients who wanted to be vaccinated had to be certified as having COPD by their respiratory physicians until mid-December 2009 in Japan, there was only a slight chance of information on vaccination being misreported.

\section{Conclusions}

We investigated the safety of the dose of influenza A (H1N1)2009 vaccines administered to COPD patients in Japan, by examining the mortality in these patients. Our study revealed no significantly increased risk of mortality after influenza A (H1N1)2009 vaccination among COPD patients. The results are, however, limited by the small sample size and low statistical power. A similar larger-scale study is needed in the future to confirm our findings.

\section{Acknowledgments}

This study was funded by a research grant from the Ministry of Health, Labour and Welfare, Japan (H22, No.61, Research on Life Science). We are grateful to Sae Imai for her excellent clerical support and to Akiko Nagai, PhD; Taichiro Tanaka MD, PhD; Naoki Kondo MD, MPH, PhD; Kohta Suzuki MD, MPH, PhD and Miri Sato, PhD, for their professional support in creating and gathering the survey sheets.

\section{References}

1. New influenza A (H1N1) virus: global epidemiological situation, June 2009 (2009). Wkly Epidemiol Rec 84: 249-257.

2. Serum cross-reactive antibody response to a novel influenza $A(H 1 N 1)$ virus after vaccination with seasonal influenza vaccine (2009). MMWR Morb Mortal Wkly Rep 58: 521-524.

3. Geier MR, Geier DA, Zahalsky AC (2003) Influenza vaccination and Guillain Barre syndrome small star, filled. Clin Immunol 107: 116-121.

4. Saito H, Endo M, Takase S, Itahara K (1980) Acute disseminated encephalomyelitis after influenza vaccination. Arch Neurol 37: 564-566.

5. (2012) Seasonal Flu Shot. CDC

6. Vellozzi C, Broder KR, Haber P, Guh A, Nguyen M, et al. (2010) Adverse events following influenza $A(H 1 N 1) 2009$ monovalent vaccines reported to the Vaccine Adverse Event Reporting System, United States, October 1, 2009-January 31, 2010. Vaccine 28: 7248-7255 
Citation: Yokomichi H, Kurihara S, Yokoyama T, Inoue E, Tanaka-Taya K, et al. (2012) Safety of the Influenza A (H1N1)2009 Vaccine in Chronic Pulmonry Obstructive Disease: A Matched Case-Control Study. J Vaccines Vaccin 3:148. doi:10.4172/2157-7560.1000148

7. Schonberger LB, Bregman DJ, Sullivan-Bolyai JZ, Keenlyside RA, Ziegler DW, et al. (1979) Guillain-Barre syndrome following vaccination in the National Influenza Immunization Program, United States, 1976--1977. Am J Epidemio 110: 105-123.

8. Ada G (2001) Vaccines and Vaccination. New England Journal of Medicine 345: 1042-1053.

9. (2010) Ministry of Health, Labour Welfare Japan.

10. (2010) Ministry of Health, Labour Welfare, Japan.

11. Rothman KJ (2002) Epidemiology: an introduction. Oxford University Press Inc New York.

12. Crockett AJ, Cranston J, Moss JR, Alpers JH (2001) A review of long-term oxygen therapy for chronic obstructive pulmonary disease. Respiratory medicine 95: 437-443.

13. Lachin JM (2011) Biostatistical Methods. (2nd edn), John Wiley \& Sons Inc, New Jersey.

14. Safety of influenza A (H1N1) 2009 monovalent vaccines - United States, October 1-November 24, 2009 (2009). MMWR Morb Mortal Wkly Rep 58: 1351-1356.

15. Liang XF, Li L, Liu DW, Li KL, Wu WD, et al. (2011) Safety of influenza A(H1N1) vaccine in postmarketing surveillance in China. N Engl J Med 364: 638-647.
16. Liang XF, Wang HQ, Wang JZ, Fang HH, Wu J, et al. (2010) Safety and immunogenicity of 2009 pandemic influenza A H1N1 vaccines in China: a multicentre, double-blind, randomised, placebo-controlled trial. Lancet 375 : 56-66.

17. Vajo Z, Tamas F, Sinka L,Jankovics I (2010) Safety and immunogenicity of a 2009 pandemic influenza A H1N1 vaccine when administered alone or simultaneously with the seasonal influenza vaccine for the 2009-10 influenza season: a multicentre, randomised controlled trial. Lancet 375: 49-55.

18. Nakada $\mathrm{H}$, Narimatsu $\mathrm{H}$, Tsubokura M, Murashige N, Matsumura T, et al. (2010) Risk of fatal adverse events after H1N1 influenza vaccination. Clin Infect Dis 50: $1548-1549$

19. McNeil MM, Broder KR, Vellozzi C,DeStefano F (2010) Risk of fatal adverse events after H1N1 influenza vaccine: limitations of passive surveillance data. Clin Infect Dis 51: 871-872; author reply 872-873.

20. Nakada H, Murashige N, Matsumura T, Kodama Y,Kami M (2010) Informa network of communication tools played an important role in sharing safety information on H1N1 influenza vaccine. Clin Infect Dis 51: 873-874

21. Simonsen L, Taylor RJ, Viboud C, Miller MA,Jackson LA (2007) Mortality benefits of influenza vaccination in elderly people: an ongoing controversy Lancet Infect Dis 7: 658-666.
Submit your next manuscript and get advantages of OMICS Group submissions

Unique features:

- User friendly/feasible website-translation of your paper to 50 world's leading languages

Audio Version of published paper

Digital articles to share and explore

Special features:

200 Open Access Journal

15,000 editorial team

21 days rapid review process

Quality and quick editorial, review and publication processing

Indexing at PubMed (partial), Scopus, DOAJ, EBSCO, Index Copernicus and Google Scholar etc

Sharing Option: Social Networking Enabled

Authors, Reviewers and Editors rewarded with online Scientific Credits

Better discount for your subsequent articles

Submit your manuscript at: www.editorialmanager.com/pharma 